\title{
O ser e o tempo do audiovisual: diálogos entre território e cultura
}

\author{
Sônia Barreto de Novaes Paschoal
}

\section{Resumo:}

Em tempos de globalização e multiculturalismo, as relações entre território e cultura tendem a diluir-se em interfaces audiovisuais configurando um espaço-tempo em que o ser local "parece" subordinado à proliferação de vozes. Assim o diálogo entre ciências e, neste caso, da ciência da informação e da comunicação faz-se imprescindível para uma compreensão dessa liquidez digital. Entendemos o audiovisual como uma instância no processo de mediação entre cultura e território, entre global e local e para tanto escolhemos Piracaia, cidade do interior paulista, como local para o exercício dessa mediação. A pesquisa-ação-colaborativa e a produção audiovisual documental sobre movimentos culturais nesta cidade incorporadas aos princípios de desenvolvimento local com base na comunicação, tecem uma economia política das narrativas de histórias.

\section{Palavras Chave:}

Territorialidade, narrativas audiovisuais; cultura; desenvolvimento local; mídias digitais.

\begin{abstract}
:
In times of globalization and multiculturalism, the relationship between territory and culture tends to be blurred in audiovisual interfaces. They organize a time-space in which the local being "seems" subordinated to the emerging voices. So the dialogue among sciences and, in this case, between information and communication sciences is indispensable to the comprehension of such digital liquidity. We understand the audiovisual as an instance of the mediation process between culture and territory, between local and global. So we have chosen Piracaia, a city in the inner region of São Paulo, as the place to practice such mediation. The research-collaborative-action and the documental audiovisual production about cultural movements in the related city joined toghether with principles of local developenment based on education have designed a political economy of the stories narratives.
\end{abstract}

\section{Keywords:}

Territoriality, audiovisual narratives, culture, local developnment, digital media.

Cada época traz seus modos de articular as coordenadas tempo-espaço e de combiná-las dentro de sistemas de representação. Com a globalização essas coordenadas se alteram profundamente e provocam efeitos múltiplos sobre o mundo cada vez mais interconectado. Esse processo de escala global atravessa fronteiras, "destruindo o espaço através do tempo" (HARVEY apud HALL, 2000: 73) e, ao interconectar comunidades desenvolve a multiplicidade de culturas, ou o multiculturalismo. Trata-se de um processo 
carregado de tensão e de negociação entre o local e o global na medida em que identidades culturais e territoriais, bem como seus vínculos, são colocadas em xeque e, ao desenvolver, dissolve culturas no mesmo limiar de fronteiras que vai da desculturação, passando pela aculturação e transculturação, como aponta Canclini para o "homem desenraizado" (CANCLINI, 2003: 109) o qual necessita se posicionar entre ser global e defender a sua identidade.

Ao resgatar memórias em Piracaia, cidade do interior paulista, por meio de recursos audiovisuais busca-se entender a construção do sujeito e do seu entormo social (1) na medida em que as memórias revelam conhecimentos geralmente não retratados pela história oficial. O sentimento de pertencimento ao lugar é nutrido e justifica ações, validando-as em níveis que vão do local ao global, do individual ao coletivo, do cotidiano ao extraordinário. Assim, as narrativas que se beneficiam do audiovisual estendem seus campos da performance corporal e, mais particularmente da voz a âmbitos outrora inimagináveis, indo de uma "simples" postagem em blogs ao desenvolvimento de redes sociais colaborativas as quais promovem mudanças substanciais de comportamento e de interações ambientais. O emprego de diferentes mídias ganha força à medida que é aplicada em uma comunidade interessada em seu desenvolvimento local e em desvelar sua vocação no contexto global.

As maneiras como os espaços se desenvolvem sob as intervenções de processos fundamentais pertencem ao povo que ali habita. Cada lugar é pensado, julgado e sentido segundo as dimensões dadas por pessoas que estão ou estavam nesse lugar de modo que este recebe um "sentimento" de centralidade. As narrativas surgem aqui como forma de alimentar este "sentimento" e o de pertencimento e, ao mesmo tempo, revelar conteúdos sobre a história local em consonância a universal.

Ao entender a história, propõe-se uma outra cotidiana cuja análise e compreensão se fazem indispensáveis ao entendimento do que as coisas e os homens são. Essas narrativas reestruturadas em mundos virtuais possibilitam identificar como os meios digitais proporcionam uma construção coletiva da memória em espaços que podem ser híbridos; em espaços de passagem ou de vivências significativas; em espaços permeados por códigos. Para tanto, estenderemos práticas e teorias em um documentário construído colaborativamente pelos protagonistas de produções culturais locais. A construção de uma rede social desenhada pelas relações entre o pesquisador e os protagonistas se faz necessária e, ao intercambiar informações, alimenta a literacia audiovisual contribuindo assim para propostas de políticas públicas voltadas ao desenvolvimento local.

\section{Proposta teórico-metodológica: o audiovisual entre cultura e território}

A fragmentação, a descontinuidade e a ruptura cultural trazidas no bojo da globalização favorecem em certo grau a proliferação de vozes, cujos sujeitos muitas vezes são desconhecidos, comprometendo o circuito de valores culturais ao deslocar sentidos de relacionamentos em cenários múltiplos. Essa tendência se tonifica ao se somar às mídias e às tecnologias de comunicação e de informação imbricados tanto no funcionamento quanto na administração da sociedade em geral. Os dispositivos que comportam essas vozes/expressões sofrem alterações na mesma proporção que provocam mudanças no ambiente. Assim entendemos o audiovisual, não como um meio ou uma ferramenta, mas como um dispositivo tecnológico que se insere como instância no diálogo entre cultura e território.

Este estudo exploratório sobre práticas e mediações audiovisuais aplicadas em Piracaia, é proposto teórico e metodologicamente sobre a tríade conceitual operacional de: cultura, território e audiovisual. A saber: 
a) A Cultura - Entendida enquanto sistema de significações (WILLIAMS, 2000) e como recurso (YÚDICE, 2006) ao desenvolvimento local e, portanto necessitada de gerência e investimentos, sejam estes nas esferas política, econômica e social. A cultura enquanto reserva disponível, aumenta a participação, o envolvimento e o constituir-se cidadão quando estes se apropriam de informações e as transformam em conhecimentos sobre o local por meio de relatos, narrativas audiovisuais e documentos históricos. As questões sobre a preservação de manifestações e objetos culturais se colocam como obra aberta (ECO, 2005) a visitações e a (re)significações, possibilitando o intercâmbio de informações culturais e históricas com outros sistemas, na medida que são disponibilizadas em rede.

b) O Território - Este se oferece, nesta pesquisa, como recorte ilustrativo de demandas geradas quando há ruptura do diálogo entre esse e a cultura. Nesse sentido, o território é um dos "[...] determinantes essenciais da identidade cultural, ao lado da constituição e da preservação de coleções. [...] é um efeito de mundo gerado pela inserção física direta, não mediada por uma representação elaborada, do indivíduo ou grupo nessa área física específica; [...] é o fato de ter o indivíduo nascido nessa área ou nela estar morando há algum tempo de modo a ter estabelecido alguma convivência com a área e seus ocupantes" (COELHO, 2004: 354, grifo do autor).

As relações estabelecidas no território, bem como o seu diálogo com a cultura serão abordados em narrativas audiovisuais, de maneira que estas venham a favorecer a apropriação daquelas e que o lugar e a memória sejam preservados e revelados por meio de sons e de imagens em movimentos articulados aos ambientes culturais globais.

c) O Audiovisual - Em consonância com o conceito trazido pela Medida Provisória 2.228-1, de 6 de setembro de 2001, entendemos o audiovisual como: "[...] produto da fixação ou transmissão de imagens, com ou sem som, que tenha a finalidade de criar a impressão de movimento, independentemente dos processos de captação, do suporte utilizado inicial ou posteriormente para fixá-las ou transmiti-las, ou dos meios utilizados para sua veiculação, reprodução, transmissão ou difusão" (BRASIL, 2001).

Entendemos as mídias digitais como dispositivos usados: i) para a observação e resgate de narrativas; ii) como um dos elementos de interpretação de uma realidade social; iii) para a mediação da realidade circunscrita nesta pesquisa e; iv) para a inserção da memória local no contexto global e vice-versa.

A tríade formada pelos conceitos de Cultura, Território e Audiovisual é articulada e ganha sustentabilidade da seguinte maneira: a cultura traz recursos à alimentação de diálogos por meio do audiovisual sobre o território. Este é percebido de uma outra maneira que, por sua vez, alimenta diálogos sobre a cultura. Essa tríade viabiliza o resgate, a produção e a circulação de conhecimentos, de bens e de serviços culturais que incidem sobre o desenvolvimento local ao se somar aos conceitos de tempo e de espaço.

Tanto o conceito de tempo quanto o de espaço são delimitados nesta pesquisa e combinados de maneira a demarcar e enriquecer os propósitos deste projeto. Assim propomos os seguintes recortes:

- O Tempo retratado nas figuras dos deuses gregos, a saber: a) Aion - o tempo do sempre, sem limites, a eternidade, a duração; b) Cronos - o tempo mensurável, que tudo consome e equilibra as ações e; c) Kairos - o tempo do significado, da contemplação, da reflexão.

- O Espaço como: a) Linguagem padrão (pattern language) - uma linguagem que se estende dos objetos sociais aos espaços, conduzindo problemas púbicos a uma inteligência cívica; b) Lugar (ALEXANDER, 1994) - enquanto sentido de pertencimento e, Não-lugar (AUGÉ, 2007) - espaços sem atribuições 
pessoais e; c) Terceiro espaço - hibridização de ambientes físicos e digitais.

Percebemos então o Tempo e o Espaço como duas tríades conceituais e operacionais que viabilizam o levantamento de informações e a construção de conhecimentos pertinentes ao processo de globalização e às questões de natureza local. Assim, justificamos o estudo exploratório sobre práticas e mediações audiovisuais aplicadas em um determinado local, neste caso, Piracaia.

\title{
Estudo de exploratório - Piracaia
}

\begin{abstract}
“À luz do celular e ouvindo a narradora contar histórias vividas naquele espaço íamos entrando na Casa (2) que fora de seus pais. A voz daquela jovem senhora povoava de sentido aquele lugar outrora significativo relacionando vidas que por ali transcorreram. Hoje a Casa está fechada sem água e sem luz, como uma amostra do que ocorre nesta e em outras cidades quando se divorciam de suas raízes culturais ou tratam-nas com indiferenças”.
\end{abstract}

Como localidade ou sede econômica (WEBER, 1971), ou como trama de rede social (LATOUR, 2005) a cidade é antes de tudo um espaço e como tal se define historicamente. Suas indagações serão sempre no sentido da sua produção e da sua formação. "A cidade é ao mesmo tempo, uma região e um lugar, porque ela é uma totalidade, e suas partes dispõem de um movimento combinado, segundo uma lei própria, que é a lei do organismo urbano, com a qual se confunde. [...]" (SANTOS, 1994: 71). O organismo urbano é tecido no cotidiano permeado de singularidades que vão conferindo ao sistema material e aos comportamentos obrigatórios (formas, concretudes, padrões e códigos) um outro modo de se relacionar ao tempo e ao espaço. Este modo está no campo das mediações que busca desvendar o mistério das formas (lugares, símbolos, significados).

As mediações audiovisuais enquanto dispositivos (3) se colocam como instâncias - porque, à medida que auxiliam nas respostas, são modificadas nas interações com o ambiente em questão, seja ele Piracaia.

Piracaia se situa a 85 quilômetros da capital paulista e conta com uma população estimada em 22.335 habitantes (4) em uma área de $385 \mathrm{~km}^{2}$. Um município predominantemente católico e com uma economia basicamente agrária. Seus poucos recursos e dispositivos culturais encontram-se ausentes e distantes nas esferas públicas, desfavorecendo o diálogo com seu entorno. Esses e outros fatores que estão sendo levantados e analisados contribuem na percepção, em Piracaia, de um crescente desinteresse de seus habitantes pelos seus bens culturais. Nesse sentido a cidade vem perdendo potências, não se reconhecendo em espaços e tempos. Há também uma dificuldade em reunir documentos e fontes sobre qualquer informação, seja ela histórica, geográfica, econômica e, dentre outras, a cultural, cujos segmentos se encontram desarticulados por falta de política pública que os compreenda.

A descoberta da vocação de uma determinada cidade está diretamente relacionada à vivência comum de seus cidadãos em seus ideais e práticas. Anulando os valores simbólicos dessas, anula-se a busca significativa, comprometendo assim o desenvolvimento local e, em outras instâncias, o global. Nesse aspecto vale ressaltar as demandas geradas pelas tecnologias e a exigência cada vez maior de competências múltiplas que geram novos desafios aos relacionamentos sociais. Por outro lado, as novas formas de ações coletivas, bem como os relacionamentos sociais requerem um potencial emancipatório 
numa inteligência cívica (ALEXANDER, 1994) que não diferencia gênero, faixa etária, raça, credo etc. As estruturas sociais são alcançadas e permeadas por engenharias, dentre elas as de softwares e de hardwares as quais, somadas às demais, perfazem as redes sociais alargando potenciais humanos, territoriais e culturais.

Dispor narrativas em redes e publicá-las em vídeos documentários são estratégias de mídia audiovisual para a formação de público, fortalecimento da imagem local e engajamento em políticas culturais, regional, nacional e internacional. Contudo, ressaltamos que o público é visto como um constituinte $a$ priori, cuja existência antecede qualquer ferramenta ou dispositivo, portanto estamos falando da essência de seres humanos portadores de visões e ideais múltiplos sobre si e sobre o mundo que ao apreenderem sua pertinência cultural constituem-se autônomos e singulares por meio do diálogo (5). Então é na prosa que se pretende dar o arranjo narrativo a contextos cujas distâncias fornecem elementos catalisadores à alimentação de repertórios e estímulo a novos percursos a partir da surpreendência cotidiana.

O resgate da memória em sons e imagens representa o enlace do ser ao tempo e ao espaço, fazendo emergir dimensões ocultas em cidades intangíveis (LATOUR, 1998).

Ao recortar tempos e espaços em narrativas (BENJAMIN, 1994) audiovisuais de episódios cotidianos vividos pela comunidade piracaiense, busca-se o resgate da liberdade interpretativa. Nessa liberdade permitida pelo diálogo se colocam encontros e desencontros de mundos, de posicionamentos diferenciados para questões comuns da vida como um acontecimento. Quais os caminhos trilhados por pessoas que vão distanciando os fazeres, as cumplicidades das histórias do universo local? As memórias (6) ocupam e consagram espaços em encontros, neste caso, encontros midiatizados. Ora o encontro é a possibilidade do "eu" em relação ao "tu" (BUBER, 1979).

O sentido de produções de narrativas está tanto na sua forma de registro quanto no modo de recuperação, resgate e difusão de tal conteúdo. As pessoas produzem, editam fazem circular suas informações (imagens/sons), elas são ao mesmo tempo produtores, divulgadores e receptores. Mas como acontece a emancipação digital em cidades cujo acesso (tendo em vista a autonomia) às tecnologias de comunicação é limitado, lembrando que: o acesso à tecnologia e o desenvolvimento cultural bem como o econômico caminham de mãos dadas.

Tais questões nos remetem a novas formas de conhecimentos construídos colaborativamente e compartilhados em telas, sejam do micro, do celular ou do vídeo e nos permite ainda, observar o que se perde e o que se ganha em memória durante esse processo, levando em consideração tempos diferenciados de contemplação e reflexão, bem como mensurar o impacto ou importância do circuito informacional para a população de Piracaia em relação ao sistema sociocultural, político e econômico. Daí a validação, as surpreendências de tais histórias (7) e o reconhecimento da autoridade de conhecimentos construídos a partir de narrativas e de informações que se atualizam ao toque dos dedos sem abandonar a complexidade na qual todos fazem história, ainda que extra-oficial.

O registro histórico da participação de pessoas e seus relatos cotidianos têm sido considerados importantes e são utilizados em bibliotecas, como por exemplo, no Programa BiblioRedes (8), o qual faz parte das Políticas Nacionais de Conteúdos Digitais do Chile; outro exemplo vem com comunidades norte-americanas e a digital storytelling (9), na qual ao se narrar histórias tira-se o foco da tecnologia para colocá-lo nas pessoas e em seus contextos históricos; no Brasil, um exemplo é o Museu da Pessoa.

Mas como indexar tais histórias estabelecendo conexões com outros portais e realidades? O estudo dos meios e processos audiovisuais sobre essa prática e a transformação desta em conhecimento requer também o olhar do cientista da informação sobre a preservação, mediação e disseminação deste 
conhecimento. Lembrando que: a geração de conteúdo local, tendo em vista o global, auxilia na apropriação da tecnologia social e que a participação na tomada de decisões, elemento de emancipação e de protagonismo, estimula o desejo singular e modifica o que é estar presente.

O uso de dispositivos tecnológicos não representa por si só a salvação para problemas sociais, culturais ou educacionais, mas somado a outras ações permitem transparências de problemas e se insinuam com possibilidades de respostas. Histórias de vidas e as memórias locais, sejam de um grupo ou de outro, intercambiam-se aos saberes globais e ao encontrar ressonâncias na literatura sobre emancipação digital se oferecem como bases objetivas sobre as quais ganham luz a reconstrução da memória amparada por meios e processos audiovisuais.

\section{Considerações finais}

Esta pesquisa-ação-colaborativa tem como objeto empírico o lugar da memória em Piracaia e a relação desta cidade com a Cidade do Conhecimento, compartilhando e colaborando com esta no desenvolvimento do conceito da Iconomia - basicamente este trata da economia da informação e dos valores dos ícones, ou ainda da economia política da imagem.

Ao relacionar as narrativas às abordagens diferenciadas, sugerimos a interseção de olhares de pesquisadores de diferentes áreas, sejam elas: estudos de cinema e audiovisual e ciência da informação, para o aprimoramento e o desenvolvimento de tecnologias e de políticas que respondam tanto a questões acadêmicas e teóricas, quanto a questões de ordem prática, social e situacional. A revisão bibliográfica constante sobre os temas relacionados será o suporte teórico tanto para reformulações como para verificação e novas conjecturas.

Nesse sentido, ao conferirmos às narrativas audiovisuais o valor resgatado sobre o ambiente adentramos também o território da formação de competências para outras ações que vão de um "simples" manusear equipamentos à construção de redes virtuais colaborativas, bem como o olhar diferenciado sobre o ambiente em que se vive. Assim, temos a oportunidade de explorar a literacia narrativa audiovisual e seus impactos nas pedagogias, nos sistemas e nas políticas culturais.

\section{Bibliografia:}

ALEXANDER, C. A pattern language: towns, buildings, construction. New York: Oxford University Press, 1977.

. Domestic architecture. Transcrição da palestra no Doors of Perception. Amsterdan, 1994. Disponível em: http://museum.doorsofperception.com/doors2/transcripts/alexan.html. Acesso em: 25/08/2010.

AUGÉ, M. Não-lugares: introdução à antropologia da supermodernidade. São Paulo: Papirus, 2007.

ARENDT, H. A condição humana. 10a. ed. Rio de Janeiro: Forense Universitária, 2008.

BENJAMIN, W. Obras escolhidas: magia e técnica, arte e política. São Paulo: Brasiliense, 1994.

BENKLER, Y. The wealth of networks. New Haven: Yale University Press, 2006.

BOSI, E. Memória e sociedade: lembranças de velhos. 13a. ed. São Paulo: Companhia das Letras, 1994. 
BRASIL. Medida provisória $\mathrm{n}^{\mathrm{o}}$ 2.228-1 de 6 de setembro de 2001. Disponível em: http://www.planalto.gov.br/ccivil_03/mpv/2228-1.htm. Acesso em: 23/09/2010.

BUBER, M. Do diálogo e do dialógico. São Paulo: Perspectiva, 2007.

. Eu e tu. 2a. ed. rev. São Paulo: Cortez \& Moraes, 1979.

CANCLINI, N. G. A globalização imaginada. São Paulo: Iluminuras, 2003.

CASTELLS, M. The informational city: information, technology, economic restructuring, and the urban regional process. Oxford: Blackwell Publishers, 1989.

COELHO, T. Dicionário crítico de política cultural: cultura e imaginário. 3a. ed. São Paulo: Iluminuras, 2004.

DENZIN, N. K. et al. O planejamento da pesquisa qualitativa: teorias e abordagens. 2a. ed. Porto Alegre: Artmed, 2006.

ECHEVERRÍA, J. Los señores del aire: telépolis y el tercer entorno. Barcelona: Destino, 1999.

ECO, U. Obra aberta: forma e indeterminações nas poéticas contemporâneas. São Paulo: Perspectiva, 2005.

HALL, S. A identidade cultural na pós-modernidade. 4a. ed. Rio de Janeiro: DP\&A, 2000.

HIGINO, A. F. F. et al. Mediação informacional no contexto universidade-sociedade-inovação: potencialidades, contradições e desafios. Belo Horizonte, v. 14, 2009. Disponível em: http://www.scielo.br/scielo.php?script=sci_arttext\&pid=S1413-99362009000400012\&lng=en\&nrm=iso.

Acesso em: 05/03/2010.

LATOUR, B. Reassembling the social: an introduction to actor-network-theory. Oxford: University Press, 2005.

LATOUR, B \& HERMANT, E. "Paris: ville invisible". Paris: La découverte-les empêcheurs de penser en rond, 1998.

LE DISPOSITIF: entre usage et concept. Hermés. Paris, n.25, set. de 1999. Disponível em: http://www.wolton.cnrs.fr/FR/hermes/ouvrages/h25.html. Acesso em: 15/09/2008.

MORSON, G. S. \& EMERSON, C. Mikhail Bakhtin: criação de uma prosaística. São Paulo: Edusp, 2008.

PERAYA, D. "Das mídias aos campus virtuais: um quadro de análise dos dispositivos de formação e de comunicação midiatizadas". Genéve: TECFA, s/d (tradução de Jairo Ferreira).

SANTOS, M. Técnica, espaço, tempo: globalização e meio técnico-científico informacional. São Paulo: Hucitec, 1994.

SCHWARTZ, G. "Laboratórios de emancipação digital: pesquisa-programa-ação em colaboratórios da Cidade do Conhecimento". In: UNIrevista. São Leopoldo, jul. ed 2006. Disponível em: http://www.unirevista.unisinos.br/_pdf/UNIrev_Schwartz.PDF. Acesso em: 08/04/2009.

SODRÉ, M. "O ethos midiatizado". In: Antropológica do espelho: por uma teoria da comunicação nãolinear e em rede. Petrópolis: Vozes, 2002.

WEBER, M. Conceitos e categorias de cidades. s/c, s/e, 1971.

WILLIAMS, R. Cultura. 2a. ed. Rio de Janeiro: Paz e Terra, 2000.

WILLIAMS, R. Palavras-chave: um vocabulário de cultura e sociedade. São Paulo: Boitempo, 2007.

YÚDICE, G. A conveniência da cultura: usos da cultura na era global. Belo Horizonte: UFMG, 2006.

\section{Notas:}

Trabalho apresentado na I Jornada Discente do Programa de Pós-Graduação em Meios e Processos Audiovisuais da Escola de Comunicações e Artes da Universidade de São Paulo (ECA-USP), realizada em novembro de 2010.

(1) Echeverría define o desenvolvimento de três entornos sociais. Primeiro entorno: espaço natural, culturas de subsistências; segundo entorno: espaço urbano, cultural e social; terceiro entorno: espaço tecnológico, deslocamento das relações do espaço público da cidade para a cidade global, à qual 
Echeverría denomina "telepolis" (ECHEVERRÍA, 1999).

(2) A Casa aqui relacionada está em desuso, mas é pretendido, e ações estão sendo voltadas neste sentido, que tal espaço venha a ser a Casa de Cultura e Memória Dirce e Eurides Badari. Esta pesquisa-ação se insere nesse contexto de maneira participativa e colaborativa.

(3) "Le dispositif s'entend, comme le rappelle le dictionnaire, dans une acception prioritairement technique, comme 'un ensemble de pièces constituant un mécanisme, un appareil quelconque'. Puis d'englober, par extension, tout agencement d'éléments humains ou matériels, réalisé en fonction d'un but àatteindre" (LE DISPOSITIF, 2008).

(4) Ver http://www.ibge.gov.br/home/estatistica/populacao/estimativa2009/POP2009_DOU.pdf.

(5) “[...] um fenômeno quase universal, a permear todo o discurso humano e todas as relações e manifestações da vida humana - em geral, tudo quanto tem sentido e significação" (BAKHTIN apud MORSON, 2008: 67).

(6) “A memória é um cabedal infinito do qual só registramos um fragmento. [...]” (BOSI, 1994: 39). Por se tratar de um conceito amplo e investigado por inúmeras ciências, tratamos o conceito de memória sob o ponto de vista social e cultural observando a literatura de autores que se dedicaram a esse tema.

(7) “Ao lado da história escrita, das datas, da descrição de períodos, há correntes do passado que só desapareceram na aparência. E que podem reviver numa rua, numa sala, em certas pessoas, como ilhas efêmeras de um estilo, de uma maneira de pensar, sentir, falar, que são resquícios de outras épocas" (BOSI, 1975: 74).

(8) Ver http://www.biblioredes.cl/bibliored/.

(9) Para Joe Lambert, segundo Higino, trata-se de "[...] um importante instrumento para o registro e o compartilhamento da história e da memória, além de uma ferramenta de apreensão da realidade social capaz de ampliar possibilidades de crítica e questionamento da ordem vigente. Sob tal prisma, ela se presta a um consistente trabalho de mediação entre universidade e inovação, pela via da responsabilidade social" (HIGINO, 2009). Nutt e Schwartz chamam a atenção da storytelling enquanto mobilização de uma economia da informação e uma forma de inclusão social. A história oral permite a inclusão social quando permite a passagem do isolamento ao diálogo. O sujeito constrói o sentido. O narrador emerge da narrativa ao construir o sentido aberto ao passado.

\section{Mini Currículo :}

Doutoranda em Meios e Processos Audiovisuais, mestre em ciência da informação e graduada em biblioteconomia e documentação pela Escola de Comunicações e Artes da Universidade de São Paulo. Pesquisadora e colaboradora no grupo Cidade do Conhecimento (CTR-ECA-USP) e ColaborI Colaboratório em Infoeducação (CBD-ECA-USP). 